\title{
Nonalcoholic Fatty Liver Disease in Children: Spectrum and Consequences
}

\author{
Aradhana Aneja ${ }^{1}$, Sadhna B Lal ${ }^{2}$
}

\begin{abstract}
Nonalcoholic fatty liver disease (NAFLD) has emerged as the commonest liver disease in children. The entire spectrum of NAFLD from simple steatosis to inflammation and fibrosis is seen in children. Pediatric NAFLD (pNAFLD) is most common in preadolescent and adolescent children and has the potential to cause severe liver-related as well as cardiovascular morbidity in young adulthood. The natural history of pNAFLD needs further elucidation. However, early diagnosis is imperative to prevent serious later outcomes. Lifestyle modification through diet and exercise remains the cornerstone in the management of pNAFLD. There is a great potential for the development of noninvasive biomarkers for diagnosis and novel pharmacotherapy to become available for the treatment of pNAFLD shortly.
\end{abstract}

Keywords: Advances in pNAFLD, Children, Nonalcoholic fatty liver disease.

Journal of Postgraduate Medicine, Education and Research (2021): 10.5005/jp-journals-10028-1429

\section{INTRODUCTION}

Nonalcoholic fatty liver disease (NAFLD), characterized by fatty infiltration of the liver, constitutes the hepatic component of obesity and metabolic syndrome (MeS). The spectrum of NAFLD encompasses simple steatosis, inflammation, and hepatocyte injury with or without fibrosis, i.e., nonalcoholic steatohepatitis (NASH), cirrhosis, as well as hepatocellular carcinoma (HCC). The current pandemic of pediatric obesity has resulted in an alarming rise in the prevalence of pediatric NAFLD (pNAFLD). This is linked to an increase of closely associated metabolic consequences, viz., prediabetes, type 2 diabetes, dyslipidemia, and hypertension. NAFLD will soon take over as the leading cause of adult liver transplantation., ${ }^{1,2}$ It is also recognized as the most common chronic liver disease in children. ${ }^{3,4}$ From the pediatric perspective, the chief concerns pertain to the natural history and morbidity caused in childhood or young adulthood. This review aims to summarize recent insights into the diagnosis, management, and consequences of pNAFLD.

\section{Definition of PNAFLD}

Pediatric NAFLD refers to a chronic fatty infiltration ( $>5 \%$ by imaging, direct quantification, or histology) of the liver of children, usually associated with midline obesity and or other components of MeS, in the absence of any genetic or metabolic disorder, steatogenic medication, malnutrition, parenteral nutrition, viral infection, or significant alcohol intake. ${ }^{5}$

\section{EPIDEMIOLOGY OF PNAFLD}

The prevalence of pNAFLD parallels that of pediatric obesity. However, though $70-90 \%$ of children with NAFLD are obese, most obese children do not have NAFLD., ${ }^{3,4}$ Mindful of the health and economic ramifications of the ongoing obesity pandemic, the World Health Organization is committed toward a goal of achieving a $0 \%$ increase in pediatric obesity by $2025 .{ }^{6} \mathrm{Globally}$, between 1975 and 2016, obesity in girls and boys of 5-19 years has increased from 0.7 to $5.6 \%$ and 0.9 to $7.8 \%$, respectively. ${ }^{7}$ For South Asia, the increase has been from $0 \%$ in both boys and girls, to $1.8 \%$ in girls and $2.6 \%$ in boys, over the same period?

\begin{abstract}
$\overline{\text { 1,2Division of Paediatric Gastroenterology, Hepatology and Nutrition, }}$ Postgraduate Institute of Medical Education and Research, Chandigarh, India

Corresponding Author: Sadhna B Lal, Division of Paediatric Gastroenterology, Hepatology and Nutrition, Postgraduate Institute of Medical Education and Research, Chandigarh, India, Phone: +91 9877302447, e-mail: sadhnalal2014@gmail.com

How to cite this article: Aneja A, Lal SB. Nonalcoholic Fatty Liver Disease in Children: Spectrum and Consequences. J Postgrad Med Edu Res 2021;55(1):39-52.

Source of support: Nil

Conflict of interest: None
\end{abstract}

An accurate assessment of the population prevalence of pNAFLD is hampered by inherent challenges: (a) The population screened, i.e., normal weight, overweight, or obese. (b) Inherent variation due to age, gender, race, and ethnicity. An elegant autopsy study of accident victims in the USA had shown a higher prevalence in adolescents, males, and Hispanics as compared to infants under 5 kids, girls, and non-Hispanics. ${ }^{4}$ (c) The screening tool used, viz., elevated alanine aminotransferase (ALT)/ultrasound (USG). USG is the less sensitive tool; however, the appropriate cut-offs for ALT are debatable, investigators having used varying cut-offs. Liver biopsy is the gold standard, but cannot be used for screening.

Thus, the projected prevalence of pNAFLD is likely to be an underestimate. The prevalence of pNAFLD in the general population was estimated to be $3-12 \%$ in normal-weight and $70-80 \%$ in obese children. ${ }^{8} \mathrm{~A}$ more recent review reported a pooled estimate across various studies to be $7.6 \%$ in the general population (5.9, 5.7, and 6.5\% in Asia, Europe, and North America) and 34.2\% in obese children. ${ }^{3}$ Cross-sectional data in Indian school children aged 5-10 years reveal a prevalence ranging from $18 \%$ in normalweight children to $25-62 \%$ in overweight and obese children. ${ }^{9-11}$

\section{Risk Factors for pNAFLD}

All obese children do not develop pNAFLD. Certain clinical risk factors have been observed to be associated with a higher 
probability of not only NAFLD but also an increased histologic severity. ${ }^{4}$ The risk factors found to be predictive of the presence of NAFLD/NASH in obese children are: ${ }^{3,4,12}$

- Severe obesity, visceral adiposity reflected by truncal obesity, adolescent males, positive family history of NAFLD or MeS, infants of obese or diabetic mothers, low birth weight with rapid catch up, insulin resistance (IR)/type 2 diabetes, ethnicity (Hispanics and Asians), hypopituitarism, syndromic obesity (Prader Willi, Alstrom's, Biedl-Bardet), raised ALT/AST/gammaglutamyl transferase (GGT), acanthosis nigricans, sedentary lifestyle, and consumption of high fructose drinks.

\section{Clinical Features and Comorbidities in PNAFLD}

\section{Symptoms}

The majority of patients with pNAFLD are asymptomatic. The problems are often more psychosocial than physical, children usually having issues of self-esteem. A minority of children may complain of right upper quadrant pain but most of the physical symptoms are nonspecific and attributable to obesity and its consequences, viz., fatigue, irritability, headache, difficulty concentration, musculoskeletal pains, symptoms related to gastroesophageal reflux, such as, regurgitation, upper abdominal pain. Bloating, functional constipation, and functional abdominal pain occur frequently in these children.

\section{Signs}

A $70-90 \%$ of the children with NAFLD are overweight/obese with body mass index (BMI) and waist circumference $>95$ th centiles. More severe grades of obesity with BMI centiles $>99$ th are more likely with raised liver enzymes. Acanthosis nigricans is present in $30-50 \%$ of patients with pNAFLD. Hepatomegaly though present is usually difficult to discern through the fat abdominal wall. Peripheral stigmata of cirrhosis are rare in pNAFLD.

\section{Comorbidities}

Children with NAFLD, especially the ones with severe obesity, have a higher incidence of comorbid conditions, such as, low bone mineral density, hypertension, dyslipidemia, obstructive sleep apnea, and polycystic ovarian disease. ${ }^{13-16}$ Interestingly, children with comorbidities tend to have a more severe histologic disease.

\section{Natural History and Outcomes of PNAFLD}

The natural history of NAFLD is well established in adults, where $50-90 \%$ of those with risk factors develop NAFLD. Of these, $70-80 \%$ have largely nonprogressive simple steatosis. ${ }^{17}$ The remaining have $\mathrm{NASH}$, two-thirds to four-fifths of whom remain stable, the remaining third developing varying degrees of fibrosis. The fibrosis worsens to cirrhosis in 5-30\% which can progress to decompensation (40-60\%), HCC (10\%), or death/liver transplantation (20-30\%). ${ }^{18}$ Liver-related mortality and morbidity significantly worsens with the worse histology.

What are the consequences of pNAFLD? Is it innocuous or are children at risk of increased morbidity and mortality? The entire histologic spectrum of pNAFLD has been reported in children, from simple steatosis to cirrhosis, the proportion varying according to whether the data are from the general population or obesity clinics. ${ }^{18}$ Accordingly, NASH/any fibrosis has been reported in
$23-85 \%$ of pNAFLD and advanced fibrosis in $0-10 \% .{ }^{18}$ It is obvious that this pool of children with NAFLD is at risk for liver-related morbidity as young adults. But how aggressive is pNAFLD as an entity? Vital questions pertaining to the natural history of pNAFLD remain unanswered due to a paucity of robust prospective longitudinal studies in children.

When does pNAFLD begin? Though the peak age for diagnosing pNAFLD is in the pre-adolescent years, there is evidence of much earlier onset. The San Diego autopsy data showed that pNAFLD existed in only $0.7 \%$ of preschoolers vis-a-vis $17.3 \%$ of 15-19 years. However, $15-26 \%$ (differing by ethnicity) of obese preschoolers had NAFLD. ${ }^{4}$ Liver steatosis possibly has a perinatal onset. Evaluation of the hepatic fat content by magnetic resonance imaging in newborn infants 1-3 weeks has shown that babies born to obese mothers with gestational diabetes or to mothers with high $\mathrm{BMI}$ had up to $68 \%$ more fat in their livers than those born to normal-weight mothers. ${ }^{19,20}$ Stillborns to mothers with diabetes were also found to have hepatic steatosis. ${ }^{21}$

\section{Does Liver Histology of pNAFLD Progress during Childhood?}

Follow-up histology of biopsy-proven pNAFLD is scarcely reported but a few investigations have addressed this issue. A-Kader et al. reported a baseline prevalence of severe steatosis in 36\%, mild and significant portal inflammation in 44 and $56 \%$, respectively, no fibrosis in $15 \%$, stage 1 , stage 2 , stage 3 fibrosis, and cirrhosis in $68,8,7$, and $2 \%$, respectively, of their cohort of 75 children with pNAFLD. Advanced fibrosis was associated with severe steatosis. Eighteen of these children were rebiopsied at a mean of 28 months: $44 \%$ showed no change in fibrosis, $39 \%$ had progression, and $6 \%$ (all had lost weight) had regression of fibrosis. ${ }^{22}$ Sixty-six children with NAFLD were followed up for 20 years and retrospectively analyzed. Severe steatosis and severe fibrosis were present in 45 and 20\%, respectively, at baseline. Fibrosis had progressed in four of the five children rebiopsied multiple times. ${ }^{23}$ The placebo arms of the TONIC (vitamin E and metformin) and CyNCh (cysteamine bitartrate delayed-release for the treatment of NAFLD in children) trials of NASH-CRN, conducted between 2005 and 2015 to study the efficacy of vitamin $\mathrm{E}$, metformin, and cysteamine bitartrate have provided some important insights. ${ }^{24,25}$ Of a total of 122 children $21 \%$ had no $\mathrm{NASH}, 47 \%$ had borderline NASH and 31\% had definite NASH, and $15 \%$ had advanced fibrosis at baseline. After a mean of 1.6 years, a progression of histology (NASH \pm fibrosis) occurred in $36 \%$ and improvement in $52 \%$. Worsening was associated with adolescent age, higher waist circumference, aminotransferases, cholesterol, and low density lipoprotein (LDL) at baseline and worsening obesity, aminotransferases, and loss of glucose homeostasis over the follow-up period. ${ }^{25}$ Hepatocellular carcinoma in pediatric NAFLD is rare, though it has been reported in the literature. ${ }^{26}$

\section{What is the Clinical Progression of pNAFLD?}

Obese children with NAFLD tend to have a high prevalence of MeS at diagnosis than obese children without NAFLD. Data from the USA, Italy, China, and Korea have shown the prevalence of MeS to range from 30 to $65 \%$ in children with NAFLD as compared to $10-15 \%$ in obese children without NAFLD. ${ }^{4,16,22-25,27}$ The increased presence of prediabetes, type II DM, hypertension, dyslipidemia, higher carotid intimal medial thickness at baseline is well documented in pNAFLD and tends to worsen if obesity persists. ${ }^{16,25,27-29}$ These markers of future cardiovascular morbidity have been associated with advanced liver fibrosis in children and thus would translate into higher liver- 
related morbidity and mortality when they are young adults. Death from decompensated liver disease and liver transplant in childhood, due to $p N A F L D$ are rare but described. ${ }^{23,27}$ Children with pNAFLD are estimated to have 14 times higher risk of dying due to liver disease. ${ }^{23}$

\section{Conclusions about the Natural History of pNAFLD}

Thus, pNAFLD seems to be a disease that may start in utero or in toddlers, though it peaks in preadolescents. Though rare, adverse clinical and histologic outcomes are possible in childhood. The prospect of pNAFLD, which by definition begins early, being more aggressive than its adult counterpart remains, though it needs to be proven by prospective follow-up of children with NAFLD as they enter adulthood. It may be inappropriate to make conclusions about the natural history of pNAFLD from adult data as the current generation is more likely to be more exposed to adverse factors in utero (maternal diabetes and IR) as well as unhealthy lifestyles causing early onset severe obesity, as compared to older generations. ${ }^{5}$

\section{Pathogenesis of pNAFLD}

Nonalcoholic fatty liver disease is a complex disease with multifaceted pathogenesis. It typically affects obese individuals with IR. The liver plays a central role in lipid and glucose metabolism and NAFLD essentially reflects impaired homeostasis of these processes. Earlier it was thought to evolve as per the double-hit hypothesis, in which the first hit was an accumulation of triglycerides in the liver followed by a second hit in which inflammatory mediators resulted in hepatocyte injury and ultimately fibrosis. Current evidence suggests multiple parallel hits that result in NAFLD by acting upon several overlapping pathways including lipotoxicity, endoplasmic reticulum (ER) stress, oxidative stress, etc.

Faulty eating habits, sedentary habits, i.e., an imbalance between calorie intake and energy expenditure, and IR cause an intrahepatic accumulation of fatty acids (FA). Insulin resistance enhances the recruitment of FA from circulation and their deposition in the liver. Fatty acids increase the vulnerability of the hepatocyte to injury and cause hepatic IR causing a vicious cycle of hepatic lipid accumulation. Fatty acids cause accumulation of incompletely oxidized substrate and ER activation. Subsequent hits involve reactive oxygen species (ROS) which cause hepatocyte damage by inhibiting mitochondrial respiratory chain enzymes and cause lipid peroxidation which damages hepatocyte membranes. A variety of insults then further damage hepatocytes, causing apoptosis, activate collagen depositing stellate cells, ultimately leading to fibrosis and cirrhosis. ${ }^{30}$

The above mechanisms are the result of a dynamic interplay or crosstalk between the liver, adipose tissue, and the gut which are affected by the combination of genetic predisposition as well as the environmental influences causing epigenetic changes. This interplay leads to a state of chronic inflammation that promotes liver injury and endothelial vascular damage. The proposed pathogenesis is depicted in Figure 1 and a brief description of the various pathogenetic factors follows.

\section{Role of Genetic Polymorphisms}

There is significant evidence for the heritability of NAFLD and even its progression to NASH and cirrhosis. The ethnic gradient with the highest prevalence in Hispanics and the least in Black Americans, ${ }^{4}$ the higher prevalence in siblings and parents (59 and 78\%) of children with NAFLD as compared to those of BMI matched children without NAFLD (17 and 37\%), ${ }^{31}$ and the 12.5-fold higher risk of progressive liver disease in patients with a family history of NAFLD cirrhosis, ${ }^{32}$ all implicate genetic variation as a key player. It is estimated that $>50 \%$ of the phenotypic variation with respect to liver fat content, inflammation, and fibrosis is accounted for by genetic polymorphisms. The early observations triggered genomewide association studies as well as single candidate gene studies which yielded an array of genetic polymorphisms, responsible for NAFLD and its progression in children. ${ }^{33}$

\section{Genetic Variants Reproducibly Associated with pNAFLD}

(1) PNPLA3: The rs738409 C > G single-nucleotide polymorphism results in the $1148 \mathrm{M}$ protein variant of patatin-like phospholipase domain-containing 3 (PNPLA3). This gene encodes for the protein adiponutrin. The $1148 \mathrm{M}$ variant results in defective lipid remodeling and causes excessive fat accumulation in the liver as well affects stellate cell function promoting inflammation and fibrogenesis. ${ }^{34}$ It has a proven role in pNAFLD. (2) TM6SF2: The rs58542926 C > $\mathrm{T}$ single-nucleotide polymorphism that gives rise to the E167K variant in transmembrane 6 superfamily member 2 (TM6SF2) is also an important contributor to fatty liver. The gene encodes the secretion of very low density lipoprotein (VLDL) and the mutation impairs VLDL secretion resulting in fat accumulation. It has also been reproducibly found to be associated with pNAFLD. ${ }^{34}$ (3) GCKR: GWAS has linked the GCKR rs1260326 polymorphism to hypertriglyceridemia, hyperglycemia, and NAFLD. The gene encodes for the glucokinase regulatory protein that binds the glucokinase protein (GK) in the nucleus, making GK inactive. The rs1260326 variant encodes for proline to leucine substitution at the 446 position ( $P 446 \mathrm{~L}$ ), resulting in a loss of the affinity of the GCKR for the GK. As a consequence, more GK is available in the cytoplasm to convert glucose to glucose 6 phosphate (G6P). The increased production of $\mathrm{G} 6 \mathrm{P}$ results in an increased rate of glycolysis, which in turn leads to increased production of malonyl-CoA, the precursor of de novo lipogenesis. This variant has been associated with NAFLD not only in adults but also in children. ${ }^{35}$

\section{Genetic Variants Less Reproducibly Associated with PNAFLD}

The rs641738 variant membrane-bound O-acyl transferase 7 (MBOAT7) gene results in altered phospholipid remodeling and contributes to the progression of NAFLD. A variant of the gene encoding protein phosphatase 1 regulatory subunit 3B (PPP1R3B) seems to have a protective effect as it shifts substrate utilization from lipogenesis to glycogenesis. Other genes thought to have an either protective or deleterious role in the progression of NAFLD to NASH and fibrosis, include variations in Mer tyrosine kinase-MERTK (protective), interferon- $\lambda$ 4-IFNL4 (deleterious), $17-\beta$ hydroxysteroid dehydrogenase $13-$ HSD17B13 (protective), apolipoprotein B-APO-B (deleterious), telomerase reverse transcriptase-TERT (deleterious). ${ }^{34}$

These genetic risk factors generally have an additive effect on fat accumulation and disease progression. Further elucidation of these genetic variations may enable risk scores for NAFLD progression to be computed, in the future, based on the pattern of genetic polymorphisms of the culprit genes found in a given individual. This may enable us to select those children who are more in need of intervention/more likely to respond to particular interventions, thus helping to tailor the treatment of pNAFLD objectively.

\section{Role of Epigenetics}

Epigenetic modifications (EM) affecting DNA methylation, histone modification, and up- or downregulation of certain miRNA's are 


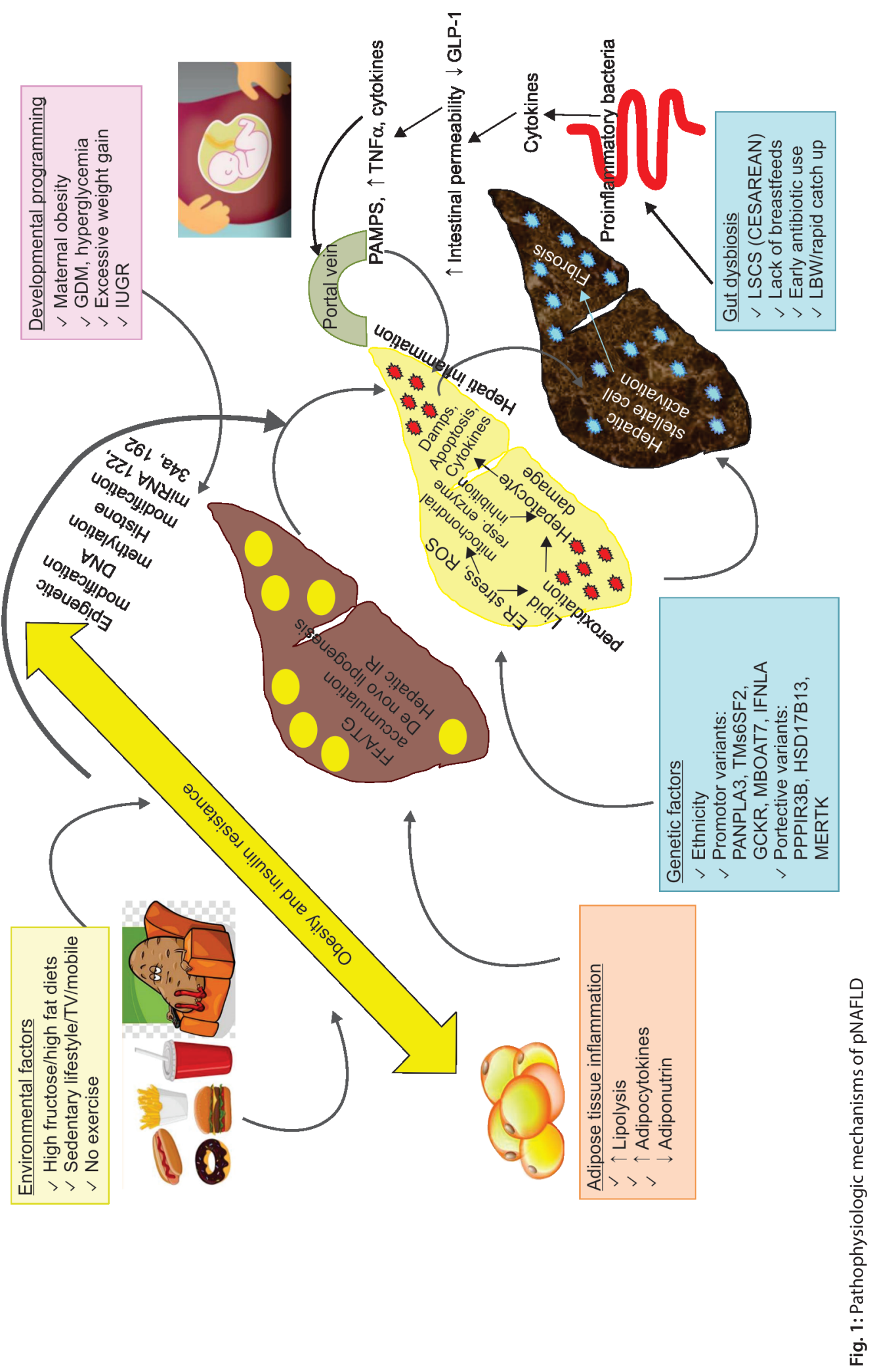


associated with NAFLD. ${ }^{30}$ These EM do not alter base sequence but affect transcription, thus affecting gene expression and phenotype. These EM are relatively stable and transmitted by cell division. Most are transient and noninheritable but some are transgenerational. These aberrations are regulated by environmental factors, e.g., diet, nutrition, stress, drugs, and intrauterine environment.

Epigenetic modification is the explanation of the effect of environmental factors on phenotype and accounts for the missing heritability of common diseases like NAFLD. In the context of NAFLD, these EMs of various genes influence lipid metabolism, ER stress, mitochondrial damage, oxidative stress, and inflammation and convert the phenotype of the liver from normal to steatosis to NASH.

DNA hypermethylation, histone modification, and miRNA changes have been detected to change the expression of certain genes playing a role in NAFLD. Simple dietary supplementation with folic acid, betaine, choline, etc., has been shown to reverse the effects of such EM in animal models. A meta-analysis published in 2018 highlighted that miR-122, miR-34a, and miR-192 are potential diagnostic markers for NAFLD and NASH with moderate diagnostic accuracy. ${ }^{36}$ The accumulation of epigenetic evidence related to NAFLD has offered a novel perspective into the pathogenesis of the disease and may contribute to identifying attractive diagnostic biomarkers and therapeutic strategies for NAFLD.

\section{Role of GUT Microbiome}

Gut microbial dysbiosis may play a role in the development and progression of NAFLD. Adult patients with NASH have intestinal microbial population enriched with ethanol-producing bacteria and have raised blood ethanol levels. Intestinal dysbiosis is associated with a state of chronic gut inflammation, altered bile acid metabolism, increased short-chain FA production of ROS, and increased intestinal permeability. It also causes altered gut hormone balance, reduction of GLP-1, and release of proinflammatory cytokines, such as, TNF-a. Delivery of bacteria, endotoxins, and cytokines (PAMP's) directly to hepatocyte toll-like receptors via the portal vein, further triggers a proinflammatory cascade and produces hepatic inflammation. ${ }^{37}$ The gut dysbiosis is also associated with poorly functioning macrophages being recruited from bone marrow, which are unable to curb the inflammatory state. ${ }^{34,37}$ In a recent study, children with NAFLD had increased intestinal dysbiosis and an abundance of genes encoding proinflammatory bacterial products as compared to children without NAFLD and less severe liver disease respectively. ${ }^{37}$

\section{Role Early Life Developmental Programming}

Maternal obesity, gestational diabetes, excessive weight gain during pregnancy, cesarean section, early life antibiotic exposure, and absence of breastfeeding have been associated with a high risk of obesity and NAFLD in the offspring, mostly via mechanisms of intestinal dysbiosis. ${ }^{34,38}$

\section{SCREENING FOR PNAFLD}

The lack of approved pharmacotherapy for NAFLD/NASH along with a lack of scientific evidence proving the benefits of screening has led the AASLD to recommend against the screening of both children and adults. ${ }^{39}$ The low sensitivity of noninvasive screening tools is an added argument in favor of this point of view. Admittedly also, due to a lack of robust data on natural history and outcomes of pNAFLD, the cost efficacy of an approach of mass screening of all obese children cannot be determined. However, the pediatric perspective differs and expert opinion is in favor of screening children for $p N A F L D$. 5,40

\section{Why Screen for pNAFLD?}

Most children with NAFLD are asymptomatic and picked up incidentally if subjected to blood chemistries or imaging for unrelated reasons. Obese children with NAFLD are at risk for disease worsening in adolescence and severe liver-related as well as cardiovascular morbidity as young adults. ${ }^{5}$ Screening of children is desirable to provide an opportunity to intervene early. ${ }^{40}$

\section{Whom to Screen for pNAFLD?}

The North American Society of Pediatric Gastroenterology, Hepatology and Nutrition (NASPGHAN) recommendations for whom to screen are: ${ }^{5}$

- All obese (BMI >95th centile) and overweight (BMI between 85th and 94th centile) children between 9 years and 11 years should be screened, if they have risk factors like central adiposity, IR, pre-diabetes or diabetes, dyslipidemia, obstructive sleep apnea, MeS, or family history of NAFLD/NASH.

- Children below 9 years may be considered for screening if they are severely obese, have hypopituitarism, or a family history of NAFLD/NASH.

- Siblings (and parents) of children proven to have NAFLD should be considered for screening (because of family clustering), especially if they also have other risk factors for NAFLD.

- If initial screening is normal, follow-up screening is recommended every 2-3 years if the risk factors remain unchanged. If there is a worsening of risk factors/appearance of fresh ones, repeat screening may be done earlier.

\section{Screening Tools for pNAFLD}

- Alanine aminotransferase estimation is the currently recommended screening tool for NAFLD, since it is cheap, simple, standardized, and available the world over. The major limitation is the determination of an appropriate cut-off. It is now believed that the standard of upper limit of normal (ULN) at $40 \mathrm{IU}$, maybe too high. In the United States, the recommendation for ULN is 22 and $26 \mathrm{IU}$, for girls and boys, respectively. ${ }^{5}$ These figures arise from large-scale populationbased surveys of healthy lean children. Appropriate cut-offs for healthy Indian children need to be investigated. Obese children with elevated ALT need lifestyle advice, providing risk factors, and symptoms are absent. However, persistent elevation $>2$ ULN, for $>3$ months, merits detailed evaluation for NAFLD and other possible etiologies. On the contrary, a marked elevation of $>80 \mathrm{IU}$, calls for urgent evaluation and management as the likelihood of severe disease is more. Elevated GGT may also be a marker of NAFLD and is often associated with worse disease. ${ }^{5}$ However, it is prudent to remember that the sensitivity of ALT is low (40-80\%) and NAFLD may exist even with normal ALT.

- Routine ultrasonographic imaging (USG) is not recommended as a screening tool due to operator subjectivity and its poor sensitivity for steatosis affecting more than one-third of the hepatocytes. Like ALT, normal sonography does not preclude NAFLD or even NASH. However, practically, USG is commonly employed as a screening tool in India. USG fat quantification may be an investigation of the future. Magnetic resonance spectroscopy is a very accurate method for liver fat quantification but is not cost-effective for screening purposes. Computed 
tomography is also a sensitive imaging technique for NAFLD but is not used in children due to the radiation risk involved.

\section{Establishing a Diagnosis of pNAFLD}

pNAFLD is a diagnosis of exclusion, requiring the presence of hepatic steatosis with the exclusion of other possible causes of not only steatosis but also of elevated enzymes. ${ }^{5}$ This is extremely pertinent in the pediatric age group as the fatty liver may be the manifestation of several inherited metabolic liver diseases as well as be a silent accompaniment of other causes of elevated enzymes (Table 1). These disorders require a different diagnostic and management approach, besides the standard lifestyle modification. The list includes common etiologies like viral hepatitis, autoimmune liver disease, and drug-induced hepatitis. Extensive workup is not required in every child, but a lack of awareness and skill to recognize these confounding problems can have serious consequences.

\section{Liver Histology and Role of Liver Biopsy}

Liver biopsy remains the gold standard for the confirmation of the diagnosis of pNAFLD, ruling out other possible etiologies and assessing the severity of NAFLD. Despite limitations of sampling error, interobserver variation, invasive nature, and risk of serious complications, it still plays a pivotal role in a subset of children with NAFLD.

\section{Liver Histology and Severity Scores}

A liver biopsy assesses four key parameters of NAFLD, viz., the severity of steatosis, inflammation, hepatocyte injury, i.e., ballooning and fibrosis. Adults with NAFLD typically tend to have type I NASH characterized by the presence of macrovesicular steatosis with ballooning degeneration and/or perisinusoidal fibrosis (zone 3 lobular involvement) and relative sparing of the portal tracts. On the contrary, the typical pediatric NASH (type II NASH) has steatosis with portal inflammation and/or fibrosis in the absence of ballooning degeneration and perisinusoidal fibrosis. ${ }^{41}$ Keeping these differences in mind, a pediatric-specific histologic severity score was developed (pediatric NAFLD histologic score) but could not be widely validated largely because a large proportion of children have features of both type I and type II NASH. ${ }^{42}$ Currently, the NAFLD Severity Score (NAS) ${ }^{43}$ is used to judge severity of pNAFLD, as it more reliably quantitates the histology across parameters of (i) Steatosis: $<5 \%$ as $0 ; 5-33 \%$ as $1 ; 33-66 \%$ as 2; and $>66 \%$ as 3 (ii) Hepatocyte ballooning: none as 0 ; few as 1; many as 2 (iii) Lobular inflammation: none as $0 ; 1-2$ foci per 20 $\times$ field as $1 ; 3-4$ foci per $20 \times$ field as $2 ;>4$ foci per $20 \times$ field as 3 . These three parameters constitute NAS. A score $<3$ rules out and $>5$ is suggestive of NASH. (iv) Fibrosis: none as 0 ; mild perisinusoidal zone 3 fibrosis as 1a; moderate perisinusoidal zone 3 fibrosis as 1b; only portal and periportal fibrosis as 1c; zone $3+$ portal/periportal fibrosis as 2; bridging fibrosis as 3; cirrhosis as 4 . The histologic scoring is mostly used in research protocols investigating the effect of pharmacotherapy.

\section{Indications of Liver biopsy in pNAFLD}

Liver biopsy is carried out in clinical settings, in the following situations (i) In children at risk for having NASH/advanced fibrosis, viz., >2 ULN ALT, AST/ALT >1, splenomegaly; (ii) Children with NAFLD and known clinical risk factors for severe diseases, like panhypopituitarism, type II DM, obstructive sleep apnea may be considered for biopsy keeping in mind the unreliability of ALT/USG; (iii) To rule out coexisting/alternative diagnostic possibilities, e.g., autoimmune hepatitis which cannot be diagnosed noninvasively.

\section{Noninvasive Assessment of pNAFLD}

The fallacies of liver biopsy and the need to objectively follow-up children with severe disease have triggered attempts to discover noninvasive markers of pNAFLD. Noninvasive diagnostics have focused on serologic biomarkers and newer imaging techniques to assess steatosis, NASH, and fibrosis.

- Biomarkers for detection of fibrosis: Several panels of biomarkers combining liver enzymes, AST/platelet ratio, hyaluronic acid, BMI, and serologic markers of fibrosis, like FIBROACTI, STEATOTEST, NAFLD Fibrosis Score, ELF, or European Liver Fibrosis Score have been used with variable success in the adult NAFLD population. ${ }^{44}$ However, these cannot be

Table 1: Differential diagnosis of pediatric fatty liver disease

\begin{tabular}{|c|c|c|c|}
\hline Inherited metabolic liver diseases & $\begin{array}{l}\text { Other rare genetic diseases/ } \\
\text { syndromes }\end{array}$ & Drugs and toxins & Systemic disorders \\
\hline - Wilson disease & - Cystic fibrosis & - Corticosteroids & - Acute starvation \\
\hline - a1 antitrypsin deficiency & $\begin{array}{l}\text { - Shwachman-Diamond } \\
\text { syndrome }\end{array}$ & - Methotrexate & - Severe protein-energy malnutrition \\
\hline - Galactosemia & $\begin{array}{l}\text { Dorfman-Chanarin } \\
\text { syndrome }\end{array}$ & - Valproic acid & - Total parenteral nutrition \\
\hline - Fructosemia & - Alstrom's syndrome & - Amiodarone & - Nonalcoholic fatty liver disease \\
\hline - Glycogen storage disorders & - Prader Willi syndrome & - Estrogens & - Polycystic ovarian disease \\
\hline - Tyrosinemia & - Bardet-Biedl syndrome & - HAART & - Hepatitis C \\
\hline - Citrin deficiency & - Cohen syndrome & - Nifedipine & - Celiac disease \\
\hline - Lysosomal acid lipase def. & - Cantu & - L-asparaginase & - Inflammatory bowel disease \\
\hline - Fatty acid oxidation defects & & $\begin{array}{l}\text { - Some antipsychotics } \\
\text { and antidepressants }\end{array}$ & - Hypothyroidism \\
\hline - Peroxisomal disorders & & & - Hypothalamic-pituitary disorders \\
\hline - Congenital lipodystrophies & & & - Nephrotic syndrome \\
\hline - Abeta/hypobetalipoproteinemia & & & - Type 1 diabetes \\
\hline - Familial hyperlipidemias & & & - Intestinal bacterial overgrowth \\
\hline - Some organic acidemias & & & - Anorexia nervosa \\
\hline
\end{tabular}


reliably applied to children, especially as their cut-offs need to be validated. The Pediatric NAFLD Fibrosis Index (PNFI) (based on age, waist circumference, and triglyceride levels) is a simple clinical index ( $>9$ rules in fibrosis; $<3$ rules out fibrosis) with a high positive predictive value for ruling in fibrosis (98.5\%), but a low negative predictive value (75\%) to exclude it. Hence, it was combined with enhanced liver fibrosis (ELF) test, which calculates a score based on serum levels of three extracellular matrix components: tissue inhibitor of metalloproteinases 1 , amino-terminal propeptide of type III procollagen, and hyaluronic acid. If PNFI is between 3 and 9, the ELF is calculated and a value of $>8.49$ predicts fibrosis in $97 \%$ with an accuracy of $87 \% .{ }^{45}$ Though promising, this test needs wider validation.

- Biomarkers for detection of NASH: In line with the pathogenesis of pNAFLD, several markers of oxidative stress, fibrosis, and apoptosis have been explored to detect severe steatosis and NASH in children.

- Adipokines are inflammatory mediators secreted by the adipose tissue. Adiponectin is a protective adipokine that suppresses proinflammatory and induces anti-inflammatory cytokines. Reduced levels are correlated with pNAFLD. Recently, Chimerin has been found to correlate with severe steatosis in pNAFLD. ${ }^{46}$ Other adipokines studied in pNAFLD are zonulin, adropin, retinol-binding protein-4, leptin, resistin, and visfatin. ${ }^{46}$

- Markers of Apoptosis are the most frequently studied in pNAFLD. These include cytokeratin 18 (CK18), soluble Fas, and soluble Fas ligand. ${ }^{34}$ Of these, estimation of the full (not caspase cleaved fragment) CK18 was found to have an area under the receiver operating characteristic curve (AUROC) of 0.80 for diagnosing NASH in pNAFLD. ${ }^{34}$

- Hepatokines are proteins almost exclusively produced by the liver. Fetuin-A, fibroblast growth factor 21 (FGF21), selenoprotein $P$, sex hormone-binding globulin (SHBG), angiopoietin-related growth factor (also known as angiopoietin-related protein 6), and leukocyte-derived chemotaxin 2 (LECT2) are considered as hepatokines possibly involved in NAFLD. Fetuin-A and FGF-21 levels are significantly increased in children with NAFLD and correlate with ALT. ${ }^{47}$

- Cytokines are produced as a result of oxidative stress. TNF-a, plasminogen activation inhibitor 1, IL-8, IL -18, soluble IL-2 receptor $a$, and Cathepsin $D$ have been studied in the context of NASH in PNAFLD. Of these, reduced levels of Cathepsin D have an impressive AUROC of 0.94 for predicting NASH and correlate with NAS. ${ }^{48}$

The search for an ideal biomarker, to replace liver biopsy, continues as the majority of the above-described markers tend to be nonspecific being also elevated in other systemic inflammatory states.

- Imaging for the detection of steatosis: The quest is for the quantification of steatosis for diagnosis and follow-ups.

- Conventional Ultrasonography: Assessment of hepatorenal echo contrast, liver parenchyma, intrahepatic vessels, and diaphragm yields a sensitivity of 75 to $90 \%$ for moderate to severe steatosis but very poor sensitivity for mild steatosis $<30 \%$. It is also not useful in severe obesity with $\mathrm{BMI}>40 .^{5}$ It may be used for follow-up but is generally used only for screening, though the NASPGHAN recommends against it.

- Controlled Attenuation Parameter (CAP) using the transient elastography (TE) device is based on the principle that fat attenuates ultrasound propagation. It is easy, reproducible, and allows fibrosis estimation at the same sitting. At a cut-off of 225 decibel/meter, the AUROC for predicting steatosis in a pediatric population was an impressive $0.93 .{ }^{49}$ Controlled attenuation parameter can also discriminate between different grades of steatosis.

- Magnetic resonance imaging (MRI), proton density fat fraction (PDFF), and HMR spectroscopy (HMRS): The mean MRI-PDFF of the liver was $2.6 \%$ for grade 0 steatosis (as defined by histology), $9.2 \%$ for grade I, $15.1 \%$ for grade II, and $26.8 \%$ for grade III. ${ }^{50}$ The AUROC for distinguishing grade I from grade II to III steatosis in children was, by PDFF was quite good at 0.87 , in children. ${ }^{51}$ However, further validation needs to be performed. HMR spectroscopy quantifies intra-hepatocyte lipids in small areas of interest. It is timeconsuming, requiring analysis after the scan, hence not widely used, though it is thought to be the most accurate imaging method for fat quantification. In children, at a cut-off of $6 \%$ HMRS had a sensitivity and specificity of 93 and $96 \%$ to discriminate the presence of fat and also showed good histologic correlation. ${ }^{52}$

- Imaging to detect fibrosis: Various types of ultrasound and MRIbased techniques have been investigated in children with good accuracy demonstrated in small studies. However, appropriate cut-offs need to be validated in other pediatric cohorts.

- Transient Elastography is easy to perform as an objective method to assess fibrosis except when severe obesity/ ascites are present. In children, the AUROC's for detecting any, significant to advanced fibrosis vary from 0.95 to 1 , and combining with PNFI detects clinically significant fibrosis with an accuracy of $98 \% .^{53,54}$

- Shear wave elastography (SWE) and acoustic radiation force impulse (ARFI) elastography can be integrated into conventional USG machines and are less affected by severe obesity and ascites compared to TE. However, they are slightly operator-dependent with an element of subjectivity vis-a-vis TE but show reproducible results. The AUROC's for discriminating mild and advanced fibrosis vary from 0.92 to 0.97 in children. ${ }^{54}$ At a cut-off of $2 \mathrm{~m} / \mathrm{second}$, ARFI was nearly $100 \%$ sensitive at discriminating mild and severe fibrosis in children. ${ }^{55}$

- Magnetic resonance elastography (MRE) is by far the most accurate noninvasive modality for liver fibrosis, unaffected by obesity, ascites, and operator skill, but uncommonly used due to the cost and a paucity of validation studies in children. The few studies in children using MRE have shown variation in AUROC's from 0.8 to 0.92 to discriminate mild from advanced fibrosis. ${ }^{56}$

A major limitation is that very few children with advanced fibrosis have been included in the above studies, so before recommending noninvasive imaging techniques for children with pNAFLD, more studies including a larger number of children with advanced fibrosis are required.

\section{MANAGEMENT OF PNAFLD}

\section{Red Flags}

Most children with NAFLD are asymptomatic and detected incidentally by ALT or sonography. Once detected, the onus is on the clinician to evaluate the child for the presence of red flag signs that might suggest underlying chronic liver disease or signs of advanced 
liver disease: the presence of jaundice, firm irregular hepatomegaly with left lobe enlargement, splenomegaly, history of variceal bleed, history or sign of coagulopathy, chronic fatigue, pruritus, features of hypersplenism (thrombocytopenia, leukopenia), persistent elevation of liver enzymes. ${ }^{5}$

\section{Additional Baseline Testing in pNAFLD}

In view of the wide differential diagnosis of pNAFLD, an additional battery of tests need to be performed at baseline in children, if they have deranged routine screening tests or a positive family history for metabolic liver diseases, as some entities if undetected can lead to serious consequences: (i) Routine Metabolic and Liver function tests: LFT: AST/ALT, INR, total protein/albumin, GGT, CBC, renal function tests, blood sugar fasting, $\mathrm{PP}, \mathrm{HbA1c}$, serum insulin, C-peptide, OGTT, lipid profile, thyroid function tests (ii) Tests for excluding other causes: Infective workup: hepatitis A IgM, hepatitis B surface $\mathrm{Ag}$, hepatitis $\mathrm{C}$ antibody, other chronic viral infections; Endocrine workup: TSH, free T4; Autoimmune liver diseases: total IgG, autoimmune markers-anti-nuclear antibody, anti-smooth muscle antibody, anti-liver kidney microsomal antibody; Celiac serology: serum transglutaminase antibody, total $\operatorname{lgA}$; Metabolic causes: serum ceruloplasmin, serum copper, 24-hour urine copper, serum lactate, blood gas, serum iron, ferritin, pyruvate, uric acid, sweat chloride test, alpha 1 antitrypsin, tandem mass spectroscopy, and urinary aminoacidogram.

The approach to a child with suspected NAFLD is given in the algorithm in Figure 2.

\section{Treatment}

There are several problems with assessing available pNAFLD treatment data, viz., a lack of standardized diagnosis, poorly defined outcomes, lack of controls and randomization, insufficient treatment and follow-up periods, and above all the uncertainty regarding the natural history of pNAFLD.

\section{pNAFLD Treatment Targets}

- Regression of NAFLD, i.e., liver steatosis, inflammation, and fibrosis.

- Resolution of NASH.

- Reduction of comorbidities predisposing to cardiovascular disease later on, viz., central adiposity, dyslipidemia, IR, hypertension, etc.

\section{Measuring pNAFLD Treatment Outcomes}

Determining the success of the various treatment modalities of pNAFLD is a difficult task. Pending the availability of reliable novel biomarkers to assess improvement, the recommendations, and suggestions are: ${ }^{5}$

- ALT: A sustained decrease by at least 10 units from baseline is a surrogate marker for improvement of inflammation and NASH. ${ }^{5}$

- MRI PDFF/MRS: Though expensive and not routinely recommended, this is the most reliable noninvasive marker for demonstration of steatosis improvement in clinical trials. ${ }^{51}$

- Liver Biopsy: This remains the gold standard for demonstrating improvement in NASH/early fibrosis which may be performed after 2-3 years of an intervention in the setting of a clinical trial. $^{5}$

- Lifestyle Modification: This is the single most important intervention in pNAFLD, focused on dietary modification and physical exercise targeted to cause weight loss. Key points that emerge are:

- It is more important to decrease central/visceral adiposity rather than subcutaneous fat. In our experience, BMI reduction may not be an ideal target as fat is often replaced by muscle. BMI reduction does not equate with fat reduction at follow-up. Therefore, it is important to reduce waist circumference. $^{34}$

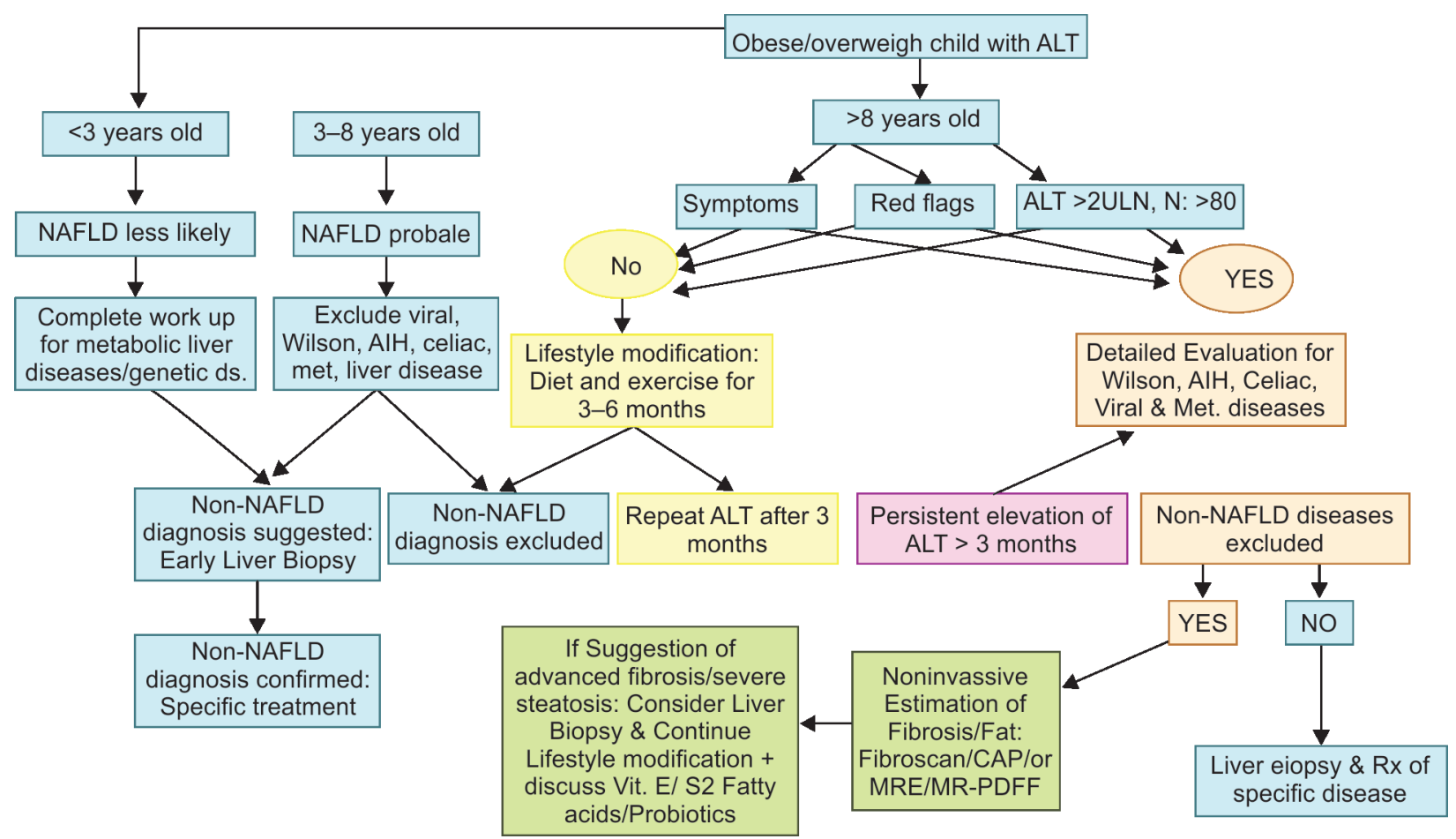

Fig. 2: Algorithmic approach to the management of pNAFLD 
- Calorie rationalization is required to optimize linear growth as well as weight. There is no difference between a low glycemic/sugar and a low-fat diet. ${ }^{5,34,56}$ General guidelines to the child and family should include advice to avoid sugary drinks (colas/juices), excess carbohydrates, junk food, discard trans-fat in favor of monounsaturated FA sources (dairy, nuts, olives, avocado, canola), ensure protective nutrients that provide natural antioxidants, ensure a diet balanced in Omega-3 (fish oil, chia seeds, flax seeds, hemp seeds) and Omega 6 (palm oil, soybean, walnuts, eggs, sunflower oil) content. $^{57}$

- Moderate- to high-intensity exercise needs to be ensured. Whereas a recent meta-analysis favored at least 3 times a week of supervised aerobic exercise, other studies have shown no difference between aerobic and resistance exercise regimes. ${ }^{58,59}$ In our set up, we have found mobile app-based exercise/workouts, mandatory outdoor time, and ensuring a TV/gadget time $<5$ hours a week is beneficial.

- It is mandatory to combine diet and exercise to achieve the desired results in pNAFLD. ${ }^{60}$ A nagging issue is of relapse as it is tough to sustain the benefit through long-term compliance in adolescents. A multidisciplinary approach followed by obesity clinics, involving the entire family in counseling, a dietician, an exercise physiologist is more effective. ${ }^{61}$

- Pharmacotherapy: The failure of lifestyle modification to caused sustained weight loss has evoked an interest in the role of pharmacotherapy for the treatment of pNAFLD. The pharmacologic treatment of pNAFLD is in the nascent stages. Candidate drugs for pNAFLD are limited and conclusions are liberally drawn from adult data. Pharmacotherapy aims to target the pathophysiologic mechanisms of NAFLD, viz., IR, dyslipidemia, oxidative stress, dysbiosis, apoptosis, fibrogenesis, etc. (Fig. 3). The drugs tried in children are discussed below.

- Vitamin E: The rationale for use is the role of oxidative stress in initiating lipid peroxidation and subsequent hepatocellular injury in NAFLD. The TONIC trial (treatment of NAFLD in children) which was a triple arm, multicenter, placebocontrolled, triple arm study, did not show any reduction of ALT as compared to placebo, after 96 weeks of treatment, though the NAS scores and ballooning were less in children with NASH who were treated with vitamin E. ${ }^{24}$ The AASLD recommends for and the NASPGHAN recommends against its use. A prudent approach would be to offer vitamin $E$ to children with biopsy-proven NASH, after discussing the cardiovascular side effects (reported in adults but absent in children after 2 years). It is imperative to combine any drug treatment with lifestyle modification.

- Cysteamine Bitartrate: This is another antioxidant that has been tested in children for 52 weeks in a double-arm placebo-controlled multicenter trial. ${ }^{62}$ There was some improvement in lobular inflammation only, the overall histologic scores being unaltered. There was, however, a reduction in ALT also. The authors observed an elevation of adiponectin, a fall of leptin levels, and CK18 fragments, with the formulation.

- Insulin sensitizers-Metformin: Metformin is the only insulin sensitizer tested in children as the third arm of the TONIC trial. ${ }^{24}$ It was not found to be better than placebo in improving either ALT or histology. However, as there was no improvement in HOMA IR also, it was thought to be a negative study due to the inadequate dose of metformin used. There are no further reports on the use of insulin sensitizers in pNAFLD.

- Polyunsaturated FA: Early studies had shown that the diet of children with NAFLD is poor in Omega- 3 fat, hence a majority of the studies have focused on it. There are reports of a sustained improvement over 2 years in liver fat content (by USG and MRI-PDFF), ALT, inflammation, and NAS reported in randomized controlled trials in children, exploring the benefit of Omega-3 FA, docosahexaenoic acid (DHA). ${ }^{63,64}$ Docosahexaenoic acid is proposed to be of benefit via an anti-inflammatory effect and a reduction of hepatic progenitor cell activation. However, another randomized control trial (RCT) reported no improvement on any of the parameters with Omega-3 supplementation, though this study used Omega- 6 sunflower oil in the placebo arm. ${ }^{65}$ Combination of DHA with eicosapentaenoic acid, vitamin $\mathrm{E}$, vitamin $D$, along with lifestyle modification is beneficial. The low cost and safety profile of PUFA make them an attractive choice for further treatment trials in pNAFLD.

- Probiotics: Gut dysbiosis has emerged as a key player in the pathogenesis of NAFLD. Manipulation of the gut microbiota by probiotics has been explored by a few double-blind RCTs in pNAFLD, with encouraging results. Lactobacillus rhamnosus GG was found to reduce ALT as well as sonographic steatosis in a cohort of obese children with raised enzymes. ${ }^{66}$ Another study randomized 48 obese children with biopsy-proven NASH, to VSL3 or placebo and found improvement of sonographic steatosis at 16 weeks, though there was no change in ALT, lipid profile, or HOMA IR. They did demonstrate an increase of GLP-1. ${ }^{67}$ A recent study randomized 68 obese children to a cocktail probiotic or placebo for 12 weeks and demonstrated improvement in ALT, triglycerides, and waist circumference. ${ }^{68}$ An important drawback of these studies is the lack of MRI and or liver biopsy to quantify improvement. However, given the safety profile of probiotics, they offer an attractive treatment modality for pNAFLD.

- Novel agents and Ongoing Pediatric trials: Some novel drugs targeting pathophysiologic mechanisms are being tested in late phase 2/phase 3 of various clinical trials and are likely to be approved for use shortly.

- Obeticholic Acid (OCA): 6 a ethyl chenodeoxycholic acid is a synthetic analog of chenodeoxycholic acid, is a ligand for the Farsenoid $X$ receptor (already approved for primary biliary cirrhosis), which modulates bile acid, lipid, and glucose homeostasis, being developed by INTERCEPT. It has demonstrated the ability to reduce IR, steatosis, and fibrosis in rodents. In a phase 2 trial (FLINT: NCT01265498, 25 mg/day $\times 72$ weeks) it led to NASH improvement in $45 \%$ patients as compared to $21 \%$ in placebo and also to greater regression of fibrosis. ${ }^{69}$ Currently, it is being tested for long-term safety and efficacy in phase 3 trials in patients with NASH (REGENERATE, ClinicalTrials.gov identifier NCT02548351: 2,370 patients) as well as in patients with fibrosis/ compensated cirrhosis (REVERSE, Clinical Trials.gov identifier NCT03439254: 540 patients). ${ }^{70}$

- Elafibrinor is a dual peroxisome proliferator-activated receptor $a$ and $\delta$ agonist (PPAR $a / \delta$ ) being developed 


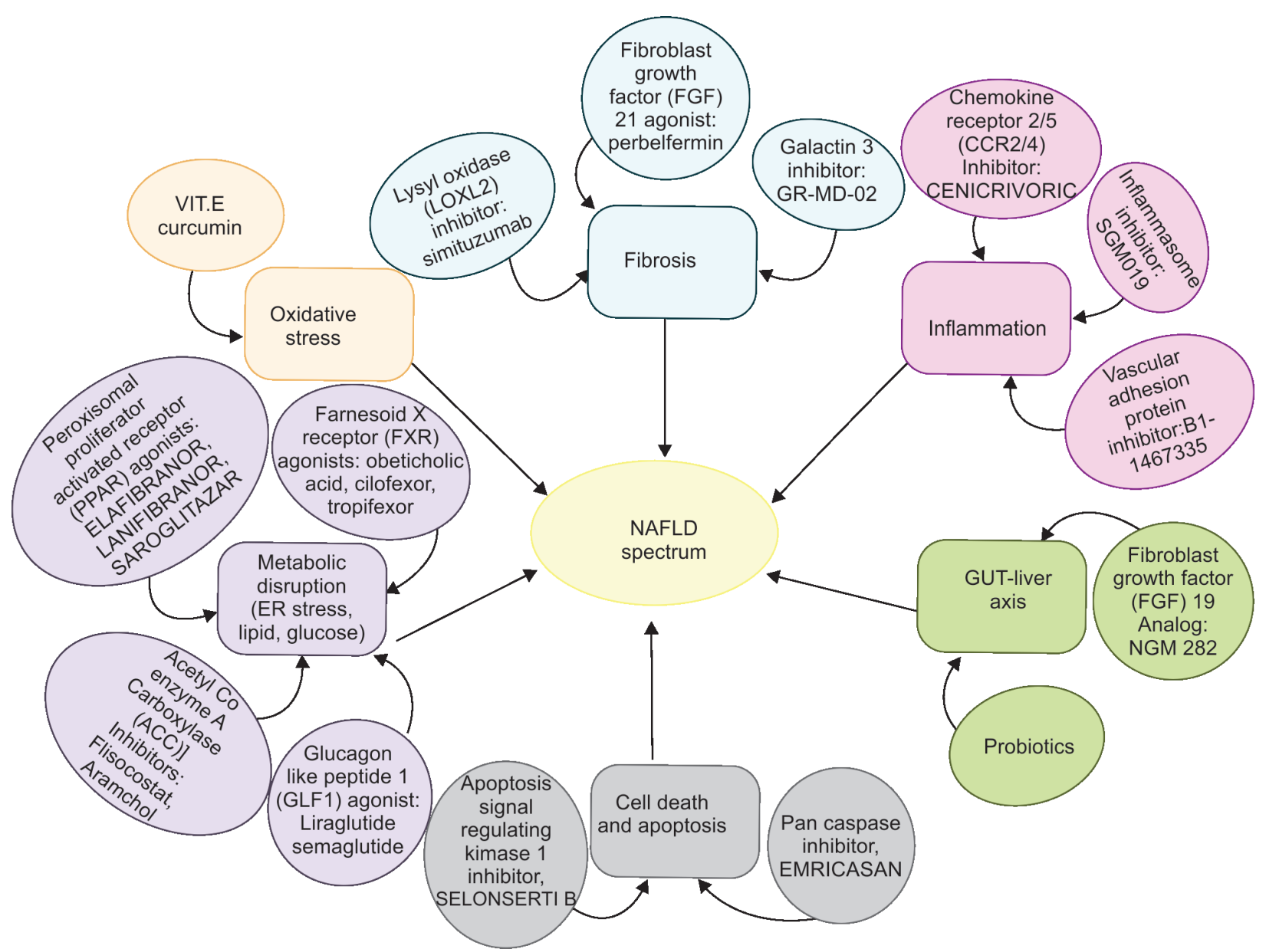

Fig. 3: Pharmacologic approaches to the management of pNAFLD

by Genfit. It is an insulin sensitizer with the potential to improve liver fat, inflammation, and fibrosis. In the phase 2b GOLDEN study (NCT01694849) at $125 \mathrm{mg} /$ day $\times 52$ weeks, it had shown significantly higher resolution of NASH without increasing fibrosis and is currently undergoing a phase 3 trial (RESOLVE-IT, NCT02704403: 2,000 patients). ${ }^{71-73}$ Other promising PPAR $a / \delta$ agonists currently under investigation are Saroglitazor (EVIDENCES IV: NCT03061721) and Lanifibranor (NATIVE: NCT03008070).

- Selonsertib: Selonsertib (GS-4997, being developed by Gilead Sciences) is an apoptosis signal-regulating kinase 1 (ASK-1) inhibitor that modulates hepatocyte apoptosis and liver fibrosis. After initial success in an open-label trial where a decrease in fibrosis was demonstrated, the drug was tried in 2 phase 3 larger-scale trials in NASH and compensated cirrhosis, with the primary outcome measure of the reduction in fibrosis by stage 1 (STELLAR 3: 880 patients NCT03053050 and STELLAR 4: 883 patients NCT03053063, respectively). Both trials were terminated in April 2019 as the primary endpoint was not reached. ${ }^{34}$ However, selonsertib remains part of a phase $2 \mathrm{~b}$ trial testing a triple combination of ACC inhibitor firsocostat and the FXR agonist cilofexor in NASH patients with fibrosis and compensated cirrhosis (ATLAS trial, NCT03449446). ${ }^{34}$
- Cenicriviroc: This is a dual CCR2/CCR5 inhibitor being developed by Allergen. These receptors are upregulated in NASH. The drug was found to inhibit macrophage infiltration and hepatic stellate cell activation in rodent models and was then successful in reducing fibrosis by stage 1 in 20 vs 10\% in placebo, after 1 year in a phase $2 b$ study (CENTAUR) ${ }^{34}$ It is currently being tested in a phase 3 study planning to recruit 2,000 patients (AURORA trial: NCT03028740). ${ }^{34}$

- Pharmacologic agents in ongoing pNAFLD trials: Several potentially therapeutic drugs tried in adults have now entered pNAFLD clinical trials:

- Aramchol is a synthetic conjugate of cholic acid and arachidic acid which inhibits stearoyl-CoA desaturase 1, the rate-limiting enzyme in MUFA synthesis thus reducing liver fat. A phase $2 \mathrm{~b}$ trial (NCT02279524) in adults testing the efficacy of 400 to $600 \mathrm{mg} / \mathrm{day}$ is underway after the success of a lower dose phase $2 a$ trial. The ARTISAN trial (Aramchol Trial to improve steatosis in Adolescent NAFLD) is planned.

- Losartan an angiotensin II receptor blocker (100 mg/day $\times 24$ weeks) has entered a phase 2 trial (NCT03042767), in 8-17-year-old children with a primary endpoint of reducing ALT. The drug improves insulin sensitivity and reduces inflammation and fibrosis by reducing 
plasminogen activator inhibitor 1 production and blocking the renin-angiotensin system. ${ }^{74}$

- Curcumin an antioxidant that can reduce inflammation is into a phase 2 trial (NCT04109742) in children.

- Empagliflozin a sodium-glucose co-transporter-2 (SGLT2) inhibitor which has been found to decrease IR has entered a phase 2 trial in children (NCT03867487).

- IMM-124E bovine colostrum enriched with antilipopolysaccharide (LPS) antibodies and can reduce the transfer of LPS from the gut and thus acts to reduce hepatic inflammation has entered a phase 2 trial in children (NCT03042767).

- Elafibranor a PPAR $a / \delta$ agonist that improves insulin sensitivity, lipid metabolism, and inflammation has also entered a phase 2 trial in children (NCT03883607).

- Bariatric surgery or weight loss surgery (WLS) is an option in severely obese adults with NAFLD, due to the frequent failure/ relapse of lifestyle modifications \pm available pharmacotherapy. Weight loss surgery leads to an improvement in liver disease due to a loss of fat mass, which affects an improvement of IR as well as systemic inflammation, an increase in the "good" adipokines, and an improvement of gut dysbiosis.

Laparoscopic sleeve gastrectomy (LSG), Roux-en-y gastric bypass, and laparoscopic adjustable gastric banding, the standard WLS in adults have been performed in adolescents but the data are scarce. An important issue is of serious complications, but as per available literature, significant complications of WLS do not occur in children.

A multicenter cohort of 242 children who underwent the above WLS for morbid obesity (BMI $>50 \mathrm{~kg} / \mathrm{m}^{2}$ ), had no postoperative complications, an average weight loss of $30 \%$ at 1 -year postoperative and $27 \%$ at 3 years, with a resolution of diabetes, pre-diabetes, hypertension, and dyslipidemia in 95, 76, 74 , and $66 \%$, respectively. ${ }^{75}$ However, $57 \%$ had iron deficiency and $13 \%$ required repeat surgery. This study clearly shows that weight loss is maintained and comorbidities in obese children do improve.

The improvement in NAFLD post-WLS, by either ALT or biopsy, in children has been shown in smaller cohorts. There was a reversal of NASH in $100 \%$ and stage 2 fibrosis in $96 \%$ in addition to a $21 \%$ weight loss and improvement in comorbidities including OSA, in severely obese children, as compared to controls, after LSG. ${ }^{76}$ However, there seems to be a low prevalence of fibrosis and NASH in children undergoing WLS, which may be due to a selection bias toward "safer" patients undergoing surgery, so the benefit of WLS on more severe liver disease in pNAFLD remains unproven. ${ }^{75,76}$ As of now WLS in pNAFLD is recommended only for severely obese children with severe comorbidities like type 2 diabetes, severe sleep apnea, or idiopathic intracranial hypertension. ${ }^{5,34}$

\section{LONG-TERM CARE IN PNAFLD}

- Children with pNAFLD are at high risk for atherosclerosis and cardiovascular morbidity as demonstrated by carotid intimamedia thickness (CIMT) by high-resolution carotid B mode ultrasonography. ${ }^{77-79}$ Carotid intima-media thickness seems to correlate with the severity of the liver disease. Hence, it is prudent to monitor for dyslipidemia and hypertension at baseline and annually thereafter.
- Children with pNAFLD must also be screened for diabetes at baseline and periodically as required with fasting blood sugar and $\mathrm{HbA}_{1} \mathrm{C}^{80}$

- Progression of liver disease using ALT, fibroscan, and repeat liver biopsy (for children in treatment trials only).

- All children with pNAFLD need to be vaccinated against hepatitis $A$ and $B$ counseled regarding the harmful effects of alcohol consumption, smoking, and nicotine use.

- Caution is advisable while using potentially hepatotoxic drugs in children with pNAFLD.

- Frequent contact with a multidisciplinary team to provide health education and reinforce lifestyle modification.

\section{Conclusion}

- pNAFLD is a leading cause of chronic liver disease in children, globally.

- The pathogenesis of pNAFLD is a complex interplay of genetic, metabolic, environmental, and microbial factors which probably become operative in utero.

- Lifestyle modification involving physical exercise and dietary changes are the only practical therapeutic interventions available for pNAFLD currently, despite limited long-term efficacy due to poor adherence.

- The discovery of genetic variants and EM and their influence on the presentation of pNAFLD may be used in the future to identify children at high risk for progressive disease.

- A better understanding of pathogenesis and its interplay with genetics will thus help to combine therapeutic agents against multiple targets in high-risk children to deliver a personalized treatment tailored to suit the individual.

- Development and validation for noninvasive biomarkers and imaging techniques, to assess and follow-up steatosis and fibrosis remains a dire need in pNAFLD. This would be invaluable to understand the natural history of pNAFLD as well as test the efficacy of therapeutic agents.

- The effect of pubertal changes on the natural history of pNAFLD needs further elucidation.

- Clinical drug trials will have to extrapolate from agents found safe in adult drug trials, to begin with. Such trials need to focus especially on the adolescent population with active pNAFLD, i.e., raised ALT, needs to have clearly defined outcomes assessed noninvasively by ALT/PDFF, and need to focus largely on drugs with metabolic targets that decrease steatosis and to a less extent on fibrosis, as the latter remains rare in childhood.

\section{References}

1. Wong RJ, Aguilar M, Cheung R, et al. Nonalcoholic steatohepatitis is the second leading etiology of liver disease among adults awaiting liver transplantation in the United States. Gastroenterology 2015;148(3):547-555. DOI: 10.1053/j.gastro.2014.11.039.

2. Cholankeril G, Ahmed A. Alcoholic liver disease replaces hepatitis $C$ virus infection as the leading Indication for liver transplantation in the United States. Clin Gastroenterol Hepatol 2018;16(8):1356-1358. DOI: 10.1016/j.cgh.2017.11.045.

3. Anderson EL, Howe LD, Jones $H E$, et al. The prevalence of nonalcoholic fatty liver disease in children and adolescents: a systematic review and meta-analysis. PLoS ONE 2015;10(10):e0140908. DOI: 10.1371/journal.pone.0140908. 
4. Schwimmer JB, Deutsch $R$, Kahen $T$, et al. Prevalence of fatty liver in children and adolescents. Pediatrics 2006;118(4):1388-1393. DOI: 10.1542/peds.2006-1212.

5. Vos MB, Abrams SH, Barlow SE, et al. NASPGHAN clinical practice guideline for the diagnosis and treatment of nonalcoholic fatty liver disease in children: recommendations from the expert committee on NAFLD (ECON) and the North American Society of Pediatric Gastroenterology, Hepatology and Nutrition (NASPGHAN). J Pediatr Gastroenterol Nutr 2017;64(2):319-334. DOI: 10.1097/ MPG.0000000000001482.

6. World Health Organization (WHO). Comprehensive Implementation Plan on Maternal, Infant and Young Child Nutrition. Geneva: WHO; 2014 https://www.who.int/nutrition/publications/CIP_document/en.

7. Di Cesare M, Sorić $M$, Bovet $P$, et al. The epidemiological burden of obesity in childhood: a worldwide epidemic requiring urgent action. BMC Med 2019;17(1):212. DOI: 10.1186/s12916-019-1449-8.

8. Alisi A, Feldstein AE, Villani A, et al. Pediatric nonalcoholic fatty liver disease: a multidisciplinary approach. Nat Rev Gastroenterol Hepatol 2012;9(3):152-161. DOI: 10.1038/nrgastro.2011.273.

9. Das MK, Bhatia V, Sibal A, et al. Prevalence of nonalcoholic fatty liver disease in normal-weight and overweight preadolescent children in Haryana, India. Indian Pediatr 2017;54(12):1012-1016. DOI: 10.1007/ s13312-017-1202-3.

10. Pawar S, Vinay Z, Choksey A, et al. Most overweight and obese Indian children have nonalcoholic fatty liver disease. Ann Hepatol 2016;15:853-861.

11. Gupta N, Jindal G, Nadda A, et al. Prevalence and risk factors for nonalcoholic fatty liver disease in obese children in rural Punjab, India. J Family Community Med 2020;27(2):103-108. DOI: 10.4103/ jfcm.JFCM_287_19.

12. Seth A, Orkin S, Yodoshi T, et al. Severe obesity is associated with liver disease severity in pediatric non-alcoholic fatty liver disease. Paediatric Obesity 2019(2):1-8. DOI: 10.1111/ijpo.12581.

13. Bloch AM, Schrag C, Faessler E, et al. Obstructive sleep apnea syndrome affects liver histology and inflammatory cell activation in pediatric nonalcoholic fatty liver disease, regardless of obesity/ insulin resistance. Am J Respir Crit Care Med 2014;189(10):66-76. DOI: 10.1164/rccm.201304-0635IM.

14. Sundaram SS, Sokol RJ, Capocelli KE, et al. Obstructive sleep apnea and hypoxemia are associated with advanced liver histology in pediatric nonalcoholic fatty liver disease. J Pediatr 2014;164(4):699. e1-706.e1. DOI: 10.1016/j.jpeds.2013.10.072.

15. Estes C, Razavi H, Loomba R, et al. Modeling the epidemic of nonalcoholic fatty liver disease demonstrates an exponential increase in burden of disease. Hepatology 2018;67(1):123-133. DOI: 10.1002/ hep.29466.

16. Selvakumar PKC, Kabbany MN, Nobili V, et al. Nonalcoholic fatty liver disease in children: hepatic and extrahepatic complications. Pediatr Clin North Am 2017;64(3):659-675. DOI: 10.1016/j.pcl.2017. 01.008.

17. Fazel $Y$, Koenig AB, Sayiner M, et al. Epidemiology and natural history of non-alcoholic fatty liver disease. Metabolism 2016;65(8):1017-1025. DOI: 10.1016/j.metabol.2016.01.012.

18. Goyal NP, Schwimmer JB. The progression and natural history of paediatric non alcoholic fatty liver disease. Clin Liver Dis 2016;20(2):325-338. DOI: 10.1016/j.cld.2015.10.003.

19. Brumbaugh $D E$, Tearse $P$, Cree-Green $M$, et al. Intrahepatic fat is increased in the neonatal offspring of obese women with gestational diabetes. J Pediatr 2013;162(5):930-936. DOI: 10.1016/j. jpeds.2012.11.017.

20. Modi N, Murgasova D, Ruager-Martin R, et al. The influence of maternal body mass index on infant adiposity and hepatic lipid content. Pediatr Res 2011;70(3):287-291. DOI: 10.1203/PDR.0b013e318225f9b1.

21. Patel KR, White FV, Deutsch GH. Hepatic steatosis is prevalent in stillborns delivered to women with diabetes mellitus. J Pediatr Gastroenterol Nutr 2015;60(2):152-158. DOI: 10.1097/ MPG.0000000000000520.
22. A-Kader HH, Henderson J, Vanhoesen $\mathrm{K}$, et al. Nonalcoholic fatty liver disease in children: a single center experience. Clin Gastroenterol Hepatol 2008;6(7):799-802. DOI: 10.1016/j.cgh.2008.03.001.

23. Feldstein $A E$, Charatcharoenwitthaya $P$, Treeprasertsuk $S$, et al. The natural history of non-alcoholic fatty liver disease in children: a follow-up study for up to 20 years. Gut 2009;58(11):1538-1544. DOI: 10.1136/gut.2008.171280.

24. Lavine JE, Schwimmer JB, Van Natta ML, et al. Effect of vitamin E or metformin for treatment of nonalcoholic fatty liver disease in children and adolescents: the TONIC randomized controlled trial. JAMA 2011;305(16):1659-1668. DOI: 10.1001/jama.2011.520.

25. Xanthakos SA, Lavine JE, Yates KP, et al. Progression of fatty liver disease in children receiving standard of care lifestyle advice. Gastroenterology 2020(5). DOI: 10.1053/j.gastro.2020.07.034.Online ahead of print.

26. Nobili V, Alisi A, Grimaldi C, et al. Non-alcoholic fatty liver disease and hepatocellular carcinoma in a 7-year-old obese boy: coincidence or comorbidity? Pediatric Obesity 2014;9(5):e99-e102. DOI: 10.1111/j.2047-6310.2013.00209.x.

27. Cioffi C, Welsh J, Cleeton R, et. al. Natural history of NAFLD diagnosed in childhood: a single-center study. Children 2017;4(5):34. DOI: 10.3390/children4050034.

28. Gökçe S, Atbinici Z, Aycan Z, et al. The relationship between pediatric nonalcoholic fatty liver disease and cardiovascular risk factors and increased risk of atherosclerosis in obese children. Pediatr Cardiol 2013;34(2):308-315. DOI: 10.1007/s00246-012-0447-9.

29. Pacifico L, Nobili V, Anania C, et al. Paediatric non alcoholic fatty liver disease, metabolic syndrome and cardiovascular risk. World J Gastroenterol 2011;17(26):3082-3091.

30. Sun C, Fan JG, Qiao L. Potential epigenetic mechanism in nonalcoholic fatty liver disease. Int J Mol Sci 2015;16(12):5161-5179. DOI: 10.3390/ijms16035161.

31. Schwimmer JB, Celedon MA, Lavine JE, et al. Heritability of nonalcoholic fatty liver disease. Gastroenterology 2009;136(5):15851592. DOI: 10.1053/j.gastro.2009.01.050.

32. Caussy C, Soni M, Cui J, et al. Nonalcoholic fatty liver disease with cirrhosis increases familial risk for advanced fibrosis. J Clin Invest 2017;127(7):2697-2704. DOI: 10.1172/JCI93465.

33. Rosaria G, Umano R, Mariangela Martino M, et al. The association between paediatric NAFLD and common genetic variants. Children 2017;4(6):49. DOI: 10.3390/children4060049.

34. Nobili V, Alisi A, Valenti L, et al. NAFLD in children: new genes, new diagnostic modalities and new drugs. Nat Rev Gastroenterol Hepatol 2019;16(9):517-530. DOI: 10.1038/s41575-019-0169-z.

35. Santoro N, Zhang CK, Zhao H, et al. Variant in the glucokinase regulatory protein (GCKR) gene is associated with fatty liver in obese children and adolescents. Hepatology 2012;55(3):781-789. DOI: 10.1002/hep.24806.

36. Liu CH, Ampuero J, Gil-Gómez A, et al. miRNAs in patients with nonalcoholic fatty liver disease: a systematic review and meta-analysis. J Hepatol 2018;69(6):1335-1348. DOI: 10.1016/j.jhep.2018.08.008.

37. Schwimmer JB, Johnson JS, Angeles JE, et al. Microbiome signatures associated with steatohepatitis and moderate to severe fibrosis in children with nonalcoholic fatty liver disease. Gastroenterology 2019;157(4):1109-1122. DOI: 10.1053/j.gastro.2019.06.028.

38. Soderborg TK, Clark SE, Mulligan CE, et al. The gut microbiota in infants of obese mothers increases inflammation and susceptibility to NAFLD. Nat Commun 2018;9(1):4462. DOI: 10.1038/s41467-01806929-0.

39. Chalasani N, Younossi Z, Lavine JE, et al. The diagnosis and management of nonalcoholic fatty liver disease: practice guidance from the American Association for the Study of Liver Diseases. Hepatology 2018;67(1):328-357. DOI: 10.1002/hep.29367.

40. Vajro P, Lenta S, Socha P, et al. Diagnosis of nonalcoholic fatty liver disease in children and adolescents: position paper of the ESPGHAN hepatology committee. JPGN 2012;54(5):700-713. DOI: 10.1097/ MPG.0b013e318252a13f. 
41. Nobili V, Alisi A, Newton KP, et al. Comparison of the phenotype and approach to pediatric vs adult patients with nonalcoholic fatty liver disease. Gastroenterology 2016;150(8):1798-1810. DOI: 10.1053/j. gastro.2016.03.009.

42. Alkhouri N, De Vito R, Alisi A, et al. Development and validation of a new histologic score for paediatric non alcoholic fatty liver disease. J Hepatol 2012;57(6):1312-1318. DOI: 10.1016/j.jhep.2012.07.027.

43. Kleiner DE, Brunt EM, Van Natta $M$, et al. Design and validation of a histologic scoring system for non alcoholic fatty liver disease. Hepatology 2005;41(6):1313-1321. DOI: 10.1002/hep.20701.

44. Castera L, Friedrich-Rust M, Loomba R. Noninvasive assessment of liver disease in patients with nonalcoholic fatty liver disease. Gastroenterology 2019;156(5):1264-1281. DOI: 10.1053/j. gastro.2018.12.036.

45. Alkhouri N, Carter-Kent C, Lopez R, et al. A combination of the pediatric NAFLD fibrosis index and enhanced liver fibrosis test identifies children with fibrosis. Clin Gastroenterol Hepatol 2011;9(2):150-155. DOI: 10.1016/j.cgh.2010.09.015.

46. Flisiak-Jackiewicz M, Lebensztejn DM. Update on pathogenesis, diagnostics and therapy of nonalcoholic fatty liver disease in children. Clin Exp Hepatol 2019;5(1):11-21. DOI: 10.5114/ceh.2019.83152.

47. Di Sessa A, Cirillo G, Guarino S, et al. . Pediatric non-alcoholic fatty liver disease: current perspectives on diagnosis and management. Pediat Health, Med Therapeut 2019;10:89-97. DOI: 10.2147/PHMT. S188989.

48. Walenbergh SMA, Houben T, Hendrikx T, et al. Plasma cathepsin D levels: a novel tool to predict pediatric hepatic inflammation. Am J Gastroenterol 2015;110(3):462-470. DOI: 10.1038/ajg.2015.29.

49. Desai NK, Harney S, Raza R, et al. Comparison of controlled attenuation parameter and liver biopsy to assess hepatic steatosis in pediatric patients. J Pediatr 2016;173:160-164. DOI: 10.1016/j. jpeds.2016.03.021.

50. Schwimmer JB, Middleton MS, Behling C, et al. Magnetic resonance imaging and liver histology as biomarkers of hepatic steatosis in children with nonalcoholic fatty liver disease. Hepatology (Baltimore, Md) 2015;61(6):1887-1895. DOI: 10.1002/hep.27666.

51. Middleton MS, Van Natta ML, Heba ER, et al. Diagnostic accuracy of magnetic resonance imaging hepatic proton density fat fraction in pediatric nonalcoholic fatty liver disease. Hepatology (Baltimore, Md) 2018;67(3):858-872. DOI: 10.1002/hep.29596.

52. Di Martino M, Pacifico L, Bezzi M, et al. Comparison of magnetic resonance spectroscopy, proton density fat fraction and histological analysis in the quantification of liver steatosis in children and adolescents. World J Gastroenterol 2016;22(39):8812-8819. DOI: 10.3748/wjg.v22.i39.8812.

53. Cho Y, Tokuhara D, Morikawa H, et al. Transient elastography-based liver profiles in a hospital-based pediatric population in Japan. PLoS ONE 2015;10(9):e0137239. DOI: 10.1371/journal.pone.0137239.

54. Mansoor S, Collyer E, Alkhouri N. A comprehensive review of noninvasive liver fibrosis tests in pediatric nonalcoholic fatty liver disease. Curr Gastroenterol Rep 2015;17(6):23. DOI: 10.1007/s11894015-0447-z.

55. Hanquinet $S$, Courvoisier D, Kanavaki A, et al. Acoustic radiation force impulse imaging-normal values of liver stiffness in healthy children. Pediatr Radiol 2013;43(5):539-544. DOI: 10.1007/s00247-0122553-5.

56. Schwimmer JB, Ugalde-Nicalo $P$, Welsh JA, et al. Effect of a low free sugar diet vs usual diet on nonalcoholic fatty liver disease in adolescent boys: a randomized clinical trial. JAMA 2019;321(3):256265.

57. Anania C,Perla FM, OliveroF, et al. Mediterranean diet and nonalcoholic fatty liver disease. World J Gastroenterol 2018;24(19):2083-2094. DOI: 10.3748/wjg.v24.i19.2083.

58. Lee $\mathrm{S}$, Bacha F, Hannon T, et al. Effects of aerobic versus resistance exercise without caloric restriction on abdominal fat, intrahepatic lipid, and insulin sensitivity in obese adolescent boys: a randomized, controlled trial. Diabetes 2012;61(11):2787-2795. DOI: 10.2337/db120214 .
59. González-Ruiz K, Ramírez-Vélez R, Correa-Bautista JE, et al. The effects of exercise on abdominal fat and liver enzymes in pediatric obesity: a systematic review and meta-analysis. Child Obes 2017;13: 272-282.

60. Mameli C, Krakauer JC, Krakauer NY, et al. Effects of a multidisciplinary weight loss intervention in overweight and obese children and adolescents: 11 years of experience. PLoS ONE 2017;12(7):e0181095. DOI: 10.1371/journal.pone.0181095.

61. van der Heijden LB, Feskens EJM, Janse A. Maintenance interventions for overweight or obesity in children: a systematic review and meta-analysis. J Obes Rev 2018;19(6):798-809. DOI: 10.1111/obr. 12664.

62. Schwimmer JB, Lavine JE, Wilson LA, et al. In children with nonalcoholic fatty liver disease, cysteamine bitartrate delayed release improves liver enzymes but does not reduce disease activity scores. Gastroenterology 2016;151(6):1141-1154. DOI: 10.1053/j. gastro.2016.08.027.

63. Nobili V, Alisi A, Della Corte C, et al. Docosahexaenoic acid for the treatment of fatty liver: randomised controlled trial in children. Nutr Metab Cardiovasc Dis 2013;23(11):1066-1070. DOI: 10.1016/j. numecd.2012.10.010.

64. Pacifico $L$, Bonci E, Di Martino $M$, et al. A double-blind, placebo-controlled randomized trial to evaluate the efficacy of docosahexaenoic acid supplementation on hepatic fat and associated cardiovascular risk factors in overweight children with nonalcoholic fatty liver disease. Nutr Metab Cardiovasc Dis 2015;25(8):734-741. DOI: 10.1016/j.numecd.2015.04.003.

65. Janczyk W, Lebensztejn D, Wierzbicka-Rucińska A, et al. Omega-3 fatty acids therapy in children with nonalcoholic fatty liver disease: a randomized controlled trial. J Pediatr 2015;166(6):1358-63.e1-3. DOI: 10.1016/j.jpeds.2015.01.056.

66. Vajro P, Mandato C, Licenziati MR, et al. Effects of lactobacillus rhamnosus strain GG in pediatric obesity-related liver disease. J Pediatr Gastroenterol Nutr 2011;52(6):740-743. DOI: 10.1097/ MPG.0b013e31821f9b85.

67. Alisi A, Bedogni G, Baviera G, et al. Randomised clinical trial: the beneficial effects of VSL\#3 in obese children with nonalcoholic steatohepatitis. Aliment Pharmacol Ther 2014;39(11): 1276-1285.

68. Famouri F, Shariat Z, Hashemipour M, et al. Effects of probiotics on nonalcoholic fatty liver disease in obese children and adolescents. J Pediatr Gastroenterol Nutr 2017;64(3):413-417. DOI: 10.1097/ MPG.0000000000001422.

69. Neuschwander-Tetri BA, Loomba R, Sanyal AJ, et al. Farnesoid X nuclear receptor ligand obeticholic acid for non-cirrhotic, nonalcoholic steatohepatitis (FLINT): a multicentre, randomised, placebocontrolled trial. Lancet (London, England) 2015;385(9972):956-965. DOI: 10.1016/S0140-6736(14)61933-4.

70. Ratziu V, Sanyal AJ, Loomba R, et al. Regenerate: Design of a pivotal, randomised, phase 3 study evaluating the safety and efficacy of obeticholic acid in patients with fibrosis due to nonalcoholic steatohepatitis. Contemp Clin Trials 2019;84:105803. DOI: 10.1016/j. cct.2019.06.017.

71. Ratziu V, Harrison SA, Francque $S$, et al. Elafibranor, an agonist of the peroxisome proliferator-activated receptor- $a$ and $-\delta$, induces resolution of nonalcoholic steatohepatitis without fibrosis worsening. Gastroenterology 2016;150(5):1147-1159.e5. DOI: 10.1053/j. gastro.2016.01.038.

72. ClinicalTrials.gov. Phase 3 study to evaluate the efficacy and safety of elafibranor versus placebo in patients with nonalcoholic steatohepatitis (NASH) (RESOLVE-IT). https://clinicaltrials.gov/ct2/ show/NCT02704403. Identifier: NCT02704403.

73. Safadi R, Konikoff FM, Mahamid M, et al. The fatty acid-bile acid conjugate aramchol reduces liver fat content in patients with nonalcoholic fatty liver disease. Clin Gastroenterol Hepatol 2014;12(12):2085-2091. DOI: 10.1016/j.cgh.2014.04.038.

74. US National Library of Medicine Clinical Trials.gov http://www. clinicaltrials.gov/ct2/show/NCT03467217(2019). 
75. Inge TH, Courcoulas AP, Jenkins TM, et al. Weight loss and health status 3 years after bariatric surgery in adolescents. N Engl J Med 2016;14(2):113-123. DOI: 10.1056/NEJMoa1506699.

76. Manco M, Mosca A, de Peppo F, et al. The benefit of sleeve gastrectomy in obese adolescents on nonalcoholic steatohepatitis and hepatic fibrosis. J Pediatr 2017;180:31-37. DOI: 10.1016/j.jpeds.2016. 08.101.

77. Baroncini LAV, Sylvestre LC, Baroncini CV, et al. Assessment of carotid intima-media thickness as an early marker of vascular damage in hypertensive children. Arq Bras Cardiol 2017;108(5):452-457. DOI: $10.5935 / a b c .20170043$
78. Rasool A, Dar W, Latief M, et al. Nonalcoholic fatty liver disease as an independent risk factor for carotid atherosclerosis. Brain Circulation 2017;3(1):35-40.

79. Zhou YY, Zhou XD, Wu SJ, et al. Nonalcoholic fatty liver disease contributes to subclinical atherosclerosis: a systematic review and meta-analysis. Hepatol Communicat 2018;2(4):376-392. DOI: 10.1002/ hep4.1155.

80. Newton KP, Hou J, Crimmins NA, et al. Prevalence of prediabetes and type 2 diabetes in children with nonalcoholic fatty liver disease. JAMA Pediatr 2016;170(10):e161971. DOI: 10.1001/jamapediatrics. 2016.1971. 